\title{
Identification of compounds with antipyretic effects and anti-endotoxin activity in different species of Lonicera japonica using spectrum-effect correlation
}

\author{
JING-XIN DING ${ }^{1}$, CHANG LIU $^{1}$, XIONG-WEI LIU ${ }^{1}$, WEI-NA YAN ${ }^{2}$, WEN-PEI LI ${ }^{3}$, \\ HUI SHI ${ }^{1}$, JIA-XIN LI ${ }^{1}$, CHENG-LIN TANG ${ }^{4}$ and YING ZHOU ${ }^{1,5}$ \\ ${ }^{1}$ School of Pharmacy, Guizhou University of Traditional Chinese Medicine; \\ ${ }^{2}$ School of Pharmaceutical Sciences; ${ }^{3}$ College of Life Sciences, Guizhou University, Guiyang, Guizhou 550025; \\ ${ }^{4}$ Guizhou Agricultural Exhibition Hall, Guiyang, Guizhou 550001; ${ }^{5}$ Guizhou Engineering Center for \\ Innovative Traditional Chinese Medicine and Ethnic Medicine, Guiyang, Guizhou 550025, P.R. China
}

Received June 11, 2020; Accepted November 19, 2020

DOI: $10.3892 / \mathrm{etm} .2021 .10097$

\begin{abstract}
Liquid chromatography (LC) is a common and straight forward approach used in the evaluation of the quality of Traditional Chinese Medicines (TCMs). Quality control is a critical step when systematically assessing the efficacy of TCMs. In the present study, the spectrum-effect correlation method was used to identify pharmacologically active substances. The aim of the present study was to investigate the underlying correlations between common chemical compounds with antipyretic effects and the anti-endotoxin activity of Lonicera japonica. The common chemical constituents of Lonicera japonica were analyzed using LC, and the antipyretic effects and anti-endotoxin activity were determined using ELISAs. Combining the results of bivariate and principal component analysis methods, eight active constituents were qualitatively and quantitatively analyzed. The results of these analyses indicated that neochlorogenic acid, chlorogenic acid, cryptochlorogenic acid and isochlorogenic acids A, B and C
\end{abstract}

Correspondence to: Professor Ying Zhou, School of Pharmacy, Guizhou University of Traditional Chinese Medicine, Dongqing South Road West, Huaxi University Town, Guiyang, Guizhou 550025, P.R. China

E-mail: yingzhou71@126.com

Abbreviations: TCM, Traditional Chinese Medicine; HPLC, high-performance liquid chromatography; LJT, Lonicera japonica Thunb.; LFH, Lonicera fulvotomentosa Hsu et S. C. Cheng; LMH, Lonicera macranthoides Hand.-Mazz.; NCA, neochlorogenic acid; CA, chlorogenic acid; CCA, cryptochlorogenic acid; SLA, secologanic acid; SL, secoxyloganin; ICAB, isochlorogenic acid B; ICAA, isochlorogenic acid A; ICAC, isochlorogenic acid C; LPS, lipopolysaccharide; AVP, arginine vasopressin; ET, endotoxin; BA, bivariate analysis; PCA, principal component analysis

Key words: Lonicera japonica, antipyretic effect, anti-endotoxin activity, spectrum-effect correlation, key substances served a synergistic role with respect to antipyretic effects and anti-endotoxin activity. The present study lays a foundation for the future clinical use of Lonicera japonica.

\section{Introduction}

Traditional Chinese Medicine (TCM) has become increasingly popular worldwide, due to its reported therapeutic effects and low toxicity. As a result, it is vital to develop novel types of TCMs and to understand the active compounds used during their clinical application (1-3). Fever is a pathological phenomenon that causes the set point of the thermoregulatory center to increase, resulting in the body producing more heat than it dissipates, thus raising the body temperature (4). Heat sources can be divided into endogenous and exogenous. Endogenous heat sources are also known as leukocyte heat sources (such as interleukins and interferons), which can act on the thermoregulatory center through the blood-brain barrier, causing the thermoregulatory point to increase. Exogenous heat sources are primarily biological macromolecules (such as proteins) that can stimulate neutrophils, eosinophils and mononuclear phagocytes to produce and release endogenous heat sources, causing the body temperature to increase $(5,6)$. The animal body is extremely sensitive to lipopolysaccharide (LPS). Even small amounts of endotoxin $(\sim 5 \mathrm{ng} / \mathrm{kg})$ can cause the body temperature to rise and fever to be maintained for $>4 \mathrm{~h}(7,8)$. LPS is a component of the outer membrane of the cell wall of Gram-negative bacteria. It is released after the death of bacteria and has a wide range of biological activities. It can act on monocytes and macrophages to produce interleukins, tumor necrosis factor and other cytokines. These cytokines act on the thermoregulatory center in the host hypothalamus, thereby increasing body temperature and causing inflammation, as well as other pathological changes (9-11).

The diversity of TCM arises from its multi-component and multi-target pharmacodynamic activity and treatment characteristics. Early research has focused on the potential antipyretic effect of compounds used in TCM and their 
underlying mechanism of action (12-18). Clarifying the efficacy and function of TCM is crucial to ensuring drug safety and quality control. The spectrum-effect correlation is studied to discern the correlation between fingerprint (chemical, biological and metabolic) with pharmacodynamic efficacy. This allows the identification of active components in TCM, and the formulation of standards to assess their internal quality (19). The spectrum-effect relationship has also been applied in multi-field research, such as single herb treatment, classic couplet medicines, compound compatibility, processing mechanism and technology optimization (20-24). In addition, it can be used to identify key substances, optimize the ratio of ingredients, improve preparation technology and track various characteristics, providing insight into novel approaches for the development of novel TCM drugs (19,25-31).

Lonicera japonica Flos is prepared from a dried flower bud or flower collected from the first harvest of Lonicera japonica blooms. Its chemical components include organic acids, flavonoids, triterpenoid saponins, iridoid terpenes, volatile oils and trace elements (32). The principal active ingredients are chlorogenic acid and luteolin, which may be quantified to ensure quality control of the preparation (33). Previous studies have reported that Lonicerae japonica Flos has numerous pharmacological effects, such as broad-spectrum antibacterial, antiviral, immunityenhancing, antioxidant, antipyretic and anti-inflammatory effects, as well as liver protection, hypoglycemia and anti-tumor activity (32,34-36). Lonicera japonica Thunb. (LJT), Lonicera fulvotomentosa Hsu et S. C. Cheng (LFH) and Lonicera macranthoides Hand.-Mazz. (LMH) represent different species of Lonicerae japonica Flos. Although their efficacy is the same, their chemical compositions differ (37).

The quality of TCM drugs has a direct impact on their efficacy (31). In the present study, LPS endotoxin was injected intraperitoneally to successfully establish a rat model of pyrotoxemia. Physiological and biochemical indexes, such as cAMP and arginine vasopressin (AVP) levels in the hypothalamus and serum endotoxin (ET) levels were measured, as previously described (38-40). The antipyretic effect and the anti-endotoxin activity of different species of Lonicera japonica were also examined. The present study provided a theoretical basis for the molecular mechanism underlying the antipyretic effects and anti-endotoxin activityof different species of Lonicera japonica and the development of safer and more effective antipyretic TCM drugs.

\section{Materials and methods}

Instruments, reagents and animals. An Agilent 1260 HPLC system (Agilent Technologies, Inc.) with a diode array detector (DAD) was used for analytical chemistry experiments and data processing. Milli-Q ultrapure water (Merck KGaA) was used for the preparation of test samples of standards. Additional instruments included the LGJ-12 Freeze dryer (Beijing Songyuan Huaxing Technology Development Co., Ltd.), the 011 Electronic thermometer (Henan Muxiang Veterinary Pharmaceutical Co. Ltd.), the 1510 microplate reader (Thermo Fisher Scientific, Inc.) and the 5415D tabletop high-speed centrifuge (Eppendorf).
LJT, LFH and LMH were collected from different villages in Guizhou (China) and identified by Professor Wei Shenghua (Guizhou University of Traditional Chinese Medicine). These samples were: i) LJT (sample no. 20170610; Suiyang, China); ii) LFH (sample no. 20170612; Qingzhen, China); and iii) LMH (sample no. 20170611; Daozhen, China). The 12 different villages of LJT were as follows: 1a-Xingtai, 1b-Xingtai, 2-Xinxiang, 3-Huaihua, 4a-Suiyang, 4b-Suiyang, 4c-Suiyang, 4d-Suiyang, 5a-Linyi, 5b-Linyi, 5c-Linyiand 6-Xiushan. The 30 different villages of LFH were as follows: 1-Caiguan, 2-Baling, 3-Maoying, 4-Yingpan, 5-Changshun, 6-Qianxi, 7-Yumo, 8-Pingba, 9a-Fengxiang, 9b-Fengxiang, 9c-Fengxiang, 10a-Yingtaowan, 10b-Yingtaowan, 11-Gantian, 12-Zhengchang, 13-Huangyangtai, 14-Yongle, 15-Yuxi, 16-Dagan, 17-Hekou, 18-Daba, 19-Zhuoshui, 20-Shichao, 21-Dejiang, 22-Sinan, 23-Dushan, 24-Tianzhu, 25a-Songtao, 25b-Songtao and 26-Xiaoguan. The 12 different villages of LMH were as follows: 1a-Xingren, 1b-Xingren, 2-Huilong, 3-Mamaya, 4-Xingyi, 5-Pengzuo, 6-Dewo, 7-Waina, 8-Hongni, 9-Zhenfeng, 10-Qingzhen and 11-Anlong.

The standards used were as follows: i) Neochlorogenic acid (NCA; cat. no. PS0601-0025); ii) chlorogenic acid (CA; cat. no. PS0131-0025); iii) cryptochlorogenic acid (CCA; cat. no. PS0775-0025); iv) secologanic acid (SLA; cat. no.PS2210-0025); v) secoxyloganin (SL; cat. no.PS2215-0020); vi) isochlorogenic acid B (ICAB; cat. no. PS0067-0025); vii) isochlorogenic acid A (ICAA; cat. no. PS0066-0025); and viii) isochlorogenic acid C (ICAC; cat. no. PS0068-0025). All standards were obtained from Chengdu Push Bio-technology Co., Ltd.). The purity of each standard was $\geq 0.98$. Acetonitrile, methanol and formic acid were of chromatographic grade, whereas the water used was ultrapure. LPS was purchased from Hefei Domei Biology Co., Ltd. (cat. no. L2880; Sigma-Aldrich; Merck KGaA). The cAMP (cat. no. LE-H3962), AVP (cat. no. LE-H1112) and ET (cat. no. LE-H8973) ELISA kits (rat) were obtained from Heifei Laier Biological Technology Co., Ltd.

Specific pathogen free Sprague-Dawley rats (age, 2 months, $\mathrm{n}=96$ ), weighing an average of $245 \pm 15 \mathrm{~g}$ (48 males and 48 females), were purchased from Chengdu Animal Health Supervision Institute. The rats were housed in a temperature-controlled room $\left(22 \pm 2^{\circ} \mathrm{C}\right)$ with $60 \pm 10 \%$ humidity on a 12 -h light/dark cycle and unrestricted access to food and water.

Preparation of the sample solution. LJT, LFH and LMH extracts were prepared using distilled water as the solvent in a 1:20 mass to volume ratio. The extracts were incubated three times at $100^{\circ} \mathrm{C}$ for 90 min each time, filtered twice, then dried at $60^{\circ} \mathrm{C}$ after concentration.

With a volume of $20 \mathrm{ml} / \mathrm{kg}$, LJT, LFH and LMH solution samples were prepared in distilled water for oral administration. The high dose was $20 \mathrm{~g} / \mathrm{kg}$ body weight (equivalent to 4 times the clinical dose), the medium dose was $10 \mathrm{~g} / \mathrm{kg}$ body weight (twice the clinical dose) and the low dose was $5 \mathrm{~g} / \mathrm{kg}$ body weight (clinical dose). With a volume of $20 \mathrm{ml} / \mathrm{kg}$ and the crude drug dosage of $6.75 \mathrm{~g} / \mathrm{kg}$, banlangen granules (Radix isatidis extract; Guangzhou Baiyunshan Pharmaceutical Holdings Co., Ltd.) was prepared for oral administration as a positive control. The blank and model groups were given equal volumes of distilled water. 
LJT, LFH and LMH solutions $(1.0 \mathrm{~g} / \mathrm{ml})$ were prepared in $50 \%$ methanol. NCA, CA, CCA, SLA, SL, ICAB, ICAA and ICAC were also dissolved in 50\% methanol. The prepared solutions were NCA $(0.75 \mathrm{mg} / \mathrm{ml}), \mathrm{CA}(1.08 \mathrm{mg} / \mathrm{ml}), \mathrm{CCA}$ $(0.72 \mathrm{mg} / \mathrm{ml})$, SLA $(0.76 \mathrm{mg} / \mathrm{ml}), \mathrm{SL}(0.52 \mathrm{mg} / \mathrm{ml}), \mathrm{ICAB}$ $(1.07 \mathrm{mg} / \mathrm{ml})$, ICAA $(0.84 \mathrm{mg} / \mathrm{ml})$ and $\operatorname{ICAC}(1.21 \mathrm{mg} / \mathrm{ml})$. Both the samples and standard solutions were filtered through a $0.45-\mu \mathrm{m}$ filter prior to analysis.

LC conditions. An Agilent Eclipse XDB-C18 column (3.9x150 mm; $5 \mu \mathrm{m}$; Agilent Technologies, Inc.) was used to analyze the samples. Data analysis was performed on an Agilent 1260 HPLC system (Agilent Technologies, Inc.) equipped with a diode array detector. The mobile phase consisted of acetonitrile (A) and $0.4 \%(v / v)$ acetic acid aqueous solution (B), and was pumped at a flow rate of $0.8 \mathrm{ml} / \mathrm{min}$. The column temperature was maintained at $35^{\circ} \mathrm{C}$. The injection volume of each sample was $10 \mu \mathrm{l}$. The chromatograms were monitored at $254 \mathrm{~nm}$. The gas was $\mathrm{N}_{2}$ and its flow rate was 1.60 SLM with the DAD.

The gradient elution program of LJT was as follows: 5-6\% A, 0-10 min; 6\% A, 10-12 min; 6-7\% A, 12-20 min; 7-8\% A, 20-25 min; 8-10\% A, 25-30 min; 10-12\% A, 30-35 min; $12-14 \%$ A, 35-40 min; $14 \%$ A, 40-45 min; $14-16 \%$ A, $45-50 \mathrm{~min} ; 16 \%$ A, 50-55 min; $16-18 \%$ A, 55-60 min; $18-20 \%$ A, 60-65 min; 20-30\% A, 65-70 min; 30-40\% A, 70-75 min; 40-60\% A, 75-80 min; 60-5\% A, 80-85 min; and 5\% A, 85-90 min.

The gradient elution program of LMH was as follows: 5-10\% A, 0-10 min; 10-15\% A, 10-20 min; $15 \%$ A, 20-25 min; 15-20\% A, 25-30 min; 20-25\% A, 30-40 min; 25-30\% A, 40-50 min; $30-40 \%$ A, 50-60 min; 40\% A, 60-70 min; 40-5\% A, 70-75 min; and 5\% A, 75-80 min.

The gradient elution program of $\mathrm{LFH}$ was as follows: 5-10\% A, 0-10 min; 10-15\% A, 10-20 min; 15\% A, 20-30 min; $15-20 \%$ A, 30-40 min; $20 \%$ A, 40-50 min; $20-30 \%$ A, 50-60 min; 30-40\% A, 60-70 min; 40\% A, 70-75 min; 40-5\% A, 75-80 min; and 5\% A, 80-85 min.

For quantitative analysis, the mobile phase was pumped at a flow rate of $1.0 \mathrm{ml} / \mathrm{min}$. The column temperature was maintained at $30^{\circ} \mathrm{C}$. In addition, the gradient elution program was the same as LMH. All other conditions were kept the same.

Fingerprint evaluation. LC fingerprints of the samples collected from different species were established and matched automatically using the Similarity Evaluation System for Chromatographic Fingerprint of TCM (version 2012; China Pharmacopoeia Committee). Furthermore, cluster analysis using SPSS (version 22; IBM Corp.) was applied to evaluate the quality of the samples from different species.

Principal component analysis (PCA). PCA is a multivariate statistical method that can retain sufficient information from data acquisition. In the present study, characteristic peaks from LC chromatograms were screened using PCA in order to identify active constituents based on spectrum-effect relationships. PCA was conducted using SPSS software (version 22.0; IBM Corp.).

Establishment of rat models of LPS toxemia. Animal experimentation was initiated following 3 days of acclimatization. The rats were divided into 12 groups (8 per group, with equal numbers of males and females). Groups 1-9 received high, medium and low doses of water extracts from LJT, LFH and LMH. The high dose was $27 \mathrm{~g} / \mathrm{kg}$ body weight (20 times the clinical dose), the medium dose was $13.5 \mathrm{~g} / \mathrm{kg}$ (10 times the clinical dose) and the low dose was $6.75 \mathrm{~g} / \mathrm{kg}$ (5 times the clinical dose). Group 10 was the fever model group, which was administered water extract by gavage daily for 6 days. Group 12 (blank) received no treatment and group 11 (positive control) received low dosage normal saline and $6.75 \mathrm{~g} / \mathrm{kg}$ Banlangen Granules, which were administered by gavage daily for 6 days. The rats were fasted overnight with free access to water prior to administration of the test solutions. On day 7, the model was considered to be established successfully when after $1 \mathrm{~h}, 100 \mu \mathrm{g} / \mathrm{kg}$ LPS endotoxin was injected into the abdominal cavity of rats in groups $1-11$. The protocol was approved by the Animal Ethical Committee of Guizhou University. All animals were treated according to the guidelines of the National Institutes of Health (41). All procedures were performed under sodium pentobarbital anesthesia (intraperitoneal injection at a dose of $50 \mathrm{mg} / \mathrm{kg}$ ).

Tissue lysate ELISA. Following anesthesia the rats were sacrificed by decapitation $6 \mathrm{~h}$ after the peak of fever. The whole brain and the hypothalamus tissue were removed quickly and placed in an ice bath. Then, $0.5 \mathrm{ml}$ cell lysis buffer (cat. no. 87792; Thermo Fisher Scientific, Inc.) was added to $50 \mathrm{mg}$ fresh hypothalamus tissue. After lysis at room temperature for $30 \mathrm{~min}$, sonication was continued for $1 \mathrm{~min}$. The samples were centrifuged $\left(3,662 \mathrm{xg} ; 15 \mathrm{~min} ; 4^{\circ} \mathrm{C}\right)$, and the supernatants were separated for testing. In each standard well, $50 \mu \mathrm{l}$ standard of different concentrations were added. In each sample well, $10 \mu \mathrm{l}$ testing sample and $40 \mu \mathrm{l}$ sample diluent were added. In blank wells, nothing was added. In all wells except the blanks, $100 \mu 1$ horseradish peroxidase (HRP) labeled detection antibody was added. cAMP (cat. no. LE-H3962), AVP (cat. no. LE-H1112) and ET (cat. no. LE-H8973) ELISA kits (rat) were obtained from Heifei Lyle Biotech Co., Ltd. The protocol was in accordance with the manufacturer's instructions. The plates were sealed with sealing film, then incubated at $37^{\circ} \mathrm{C}$ for $1 \mathrm{~h}$. After discarding the excess liquid, each well was filled with detergent five times for 1 min each time. In each well, $50 \mu \mathrm{l}$ substrate $\mathrm{A}$ and $50 \mu \mathrm{l}$ substrate $\mathrm{B}$ were added to each well, and the plates were incubated at $37^{\circ} \mathrm{C}$ in the dark for $15 \mathrm{~min}$. After incubation, $50 \mu \mathrm{l}$ termination solution was added to each well. After $15 \mathrm{~min}$ at $37^{\circ} \mathrm{C}$, the optical density (OD) value of each well was measured using a 1510 microplate reader at $450 \mathrm{~nm}$. A standard curve was drawn using the OD values obtained from the standards. According to this curve equation, the concentration of each sample was calculated.

Serum ELISA. Rats were anesthetized with sodium pentobarbital $6 \mathrm{~h}$ after the peak of fever, and $5 \mathrm{ml}$ blood was obtained from the abdominal aorta. The samples were centrifuged (845 x g; $30 \mathrm{~min}, 4^{\circ} \mathrm{C}$ ) in order to obtain serum for testing. The concentrations of ET (cat. no. LE-H8973) in serum samples was determined using a double antibody sandwich ELISA, for which the determination method was the same as that of cAMP (cat. no. LE-H3962) and AVP (cat. no. LE-H1112). All kits were obtained from Heifei Lyle Biotech Co., Ltd. 

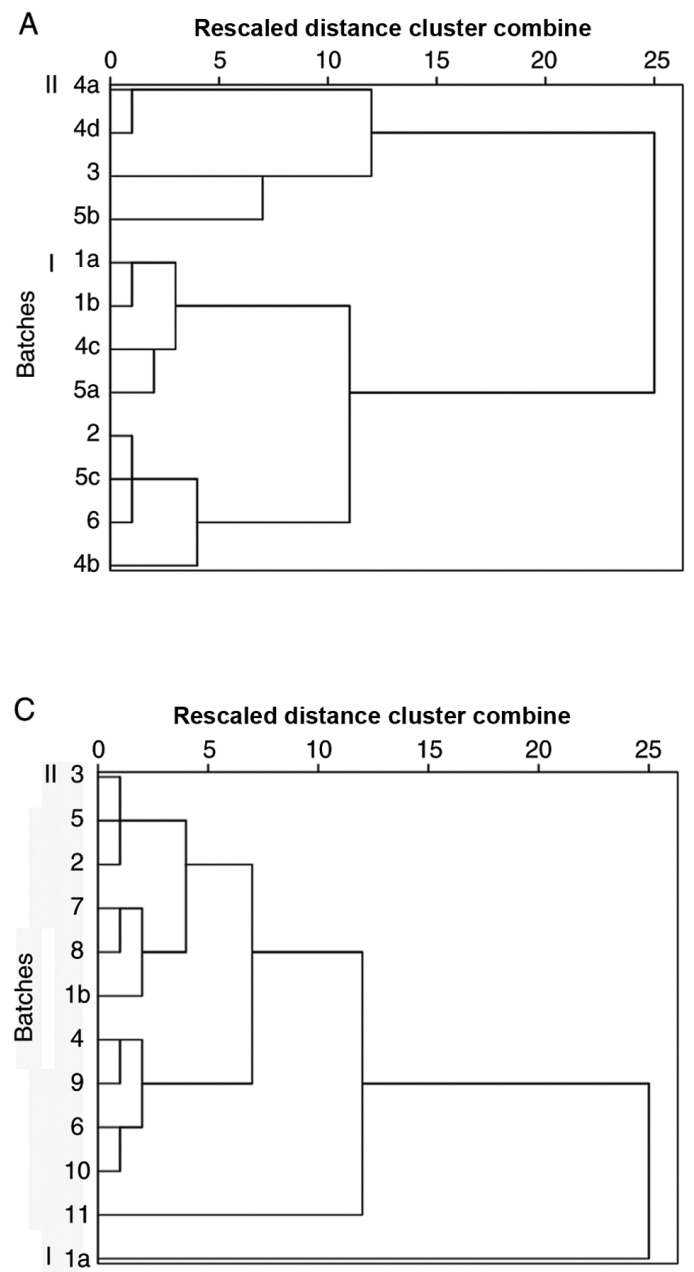

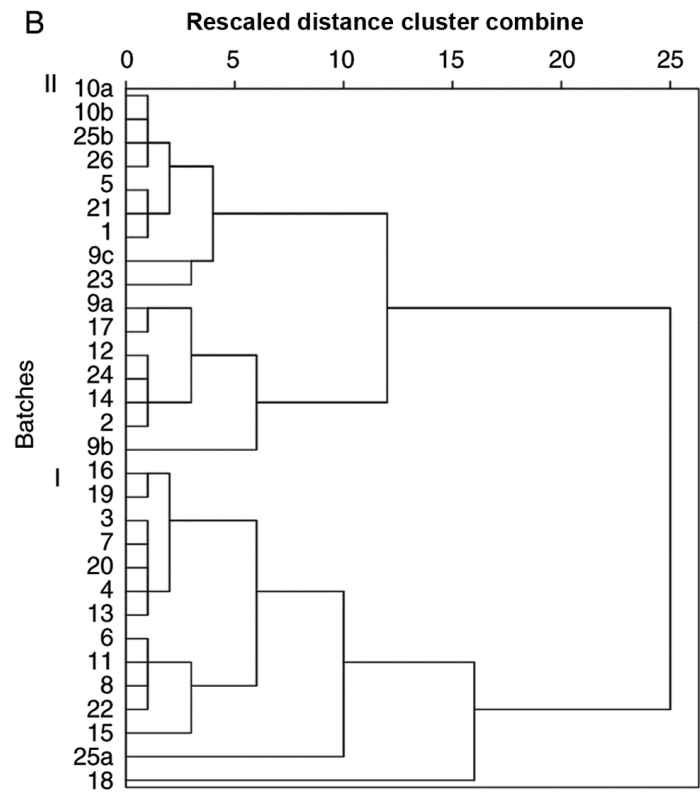

Figure 1. Cluster analysis of LJT, LFH and LMH from different batches. (A) Cluster analysis of LJT. from 12 different batches. (B) Cluster analysis of LFH from 30 different batches. (C) Cluster analysis of LMH. from 12 different batches. LJT, Lonicera japonica Thunb; LFH, Lonicera fulvotomentosa Hsu; LMH, Lonicera macranthoides Hand-Mazz.

Statistical analysis. Two-tailed paired t-tests and one-way ANOVA followed by Tukey's post hoc test were used to identify statistically significant between groups. $\mathrm{P}<0.05(95 \%$ confidence interval) was considered to indicate a statistically significant difference. Pearson's correlation coefficient was used to calculate the relative peak areas, which corresponded with antipyretic effect and anti-endotoxin activity of the samples. Correlation coefficients $>0.3$ were considered significant $(\mathrm{P}<0.05)$, whereas correlation coefficients $>0.5$ were considered highly significantly $(\mathrm{P}<0.01)$. PCA was used to evaluate characteristic peak areas in the chromatograms of the samples from different species. Bivariate analysis was used to assess the correlation of peak areas with their antipyretic effect and anti-endotoxin activity. Statistical analysis was conducted using SPSS software (version 22.0; IBM Corp.). Data are presented as the mean \pm SD and 6 experimental repeats of each test were performed.

\section{Results}

Establishment and evaluation of fingerprint. The relative standard deviation of the retention time and peak area of characteristic peaks were 0.041 and $0.082 \%$ for precision, 0.390 and $0.173 \%$ for reproducibility, and 0.098 and $0.103 \%$ for stability. The similaritiesof the chromatograms were all $>0.9$. These findings indicated that the present LC method of fingerprint establishment was reliable.

Cluster analysis. The results of cluster analysis are presented in Fig. 1. The 12 different batches of LJT were divided into two categories. The first category consisted of 4b, 6, 5c, 2, 5a, $4 \mathrm{c}, 1 \mathrm{~b}$ and $1 \mathrm{a}$. The second category consisted of $5 \mathrm{~b}, 3,4 \mathrm{~d}$ and 4a. The 30 different batches of LFH were divided into two categories. The first category consisted of 18, 25, 15, 22, 8, 11, $6,13,4,20,7,3,19$ and 16 . The second category comprised $9 \mathrm{~b}$, $2,14,24,12,17,9 \mathrm{a}, 23,9 \mathrm{c}, 1,21,5,26,25 \mathrm{~b}, 10 \mathrm{~b}$ and $10 \mathrm{a}$. The 12 different batches of LMH were divided into two categories as follows: i) a; and ii) 11, 10, 6, 9, 4, 1b, 8, 7, 2, 5 and 3. Although they were clustered into two categories, the differences were not significant, which indicated that there was no marked difference between different species of LJT, LFH and LMH.

Qualitative and quantitative analysis of the eight main chemical constituents. Under quantitative analysis conditions, the linear regression equations for NCA, CA, CCA, SLA, SL, ICAB, ICA and ICAC were as follows: i) NCA, Y=1104.2X+26.119 $(\mathrm{r}=1)$ in the concentration range of $1.50-13.50 \mu \mathrm{g} / \mathrm{ml}$; ii) $\mathrm{CA}, \mathrm{Y}=1105.4 \mathrm{X}+15.676(\mathrm{r}=1)$ in the concentration range 
of $2.59-17.28 \mu \mathrm{g} / \mathrm{ml}$; iii) CCA, $\mathrm{Y}=973.46 \mathrm{X}+5.8348(\mathrm{r}=1)$ in the concentration range of $0.72-12.96 \mu \mathrm{g} / \mathrm{ml}$; iv) SLA, $\mathrm{Y}=598.22 \mathrm{X}+28.842(\mathrm{r}=0.9998)$ in the concentration range of $0.76-12.96 \mu \mathrm{g} / \mathrm{ml}$; v) SL, Y=509.85X-2.865 ( $\mathrm{r}=1)$ in the concentration range of 1.04-9.36 $\mu \mathrm{g} / \mathrm{ml}$; vi) $\mathrm{ICAB}, \mathrm{Y}=1277.9 \mathrm{X}+2.5534$ $(\mathrm{r}=1)$ in the concentration range of $2.14-19.26 \mu \mathrm{g} / \mathrm{ml}$; vii) ICAA, $\mathrm{Y}=1169.4 \mathrm{X}-1.0839(\mathrm{r}=1)$ in the concentration range of $0.84-15.12 \mu \mathrm{g} / \mathrm{ml}$; and viii) ICAC, $\mathrm{Y}=1588.8 \mathrm{X}+8.6825(\mathrm{r}=1)$ in the concentration range of $2.24-21.78 \mu \mathrm{g} / \mathrm{ml}$. These results all indicated a linear relationship (Table I). The structures of eight main chemical constituents identified in different species of LJT, LFH and LMH are presented in Fig. 2.

Determination of the antipyretic effect and anti-endotoxin activity. There was a highly significant difference in body temperature between the normal groups and the model control group (Table II), indicating that the rat endotoxin model was successfully established. Compared with the model group, there was significant difference in antipyretic effect in each experimental group. The extract significantly inhibited the increase in CAMP and its secretion, thus reducing the heat output of the body. Compared with the model group, there were highly significant differences in the experimental groups 2, 3, 5, 6, 7, 8 and 9 (Table II). This indicated that the extract promoted an increase in the levels of the antidiuretic hormone AVP to different degrees. Compared with the model group, there was a highly significant difference in all 9 experimental groups. Thus, the extract had an anti-endotoxin effect in vivo.

$P C A$. As presented in Table III, the eigenvalues of the first two principal components were $>1$, and the contribution rate of the first principal component was $67.447 \%$, indicating that the first principal componentwas able to explain $67.447 \%$ of the antipyretic effect of different species of LJT, LFH and LMH. The second principal component could explain $32.553 \%$, and the cumulative contribution rate of the first two principal components was $100 \%$. Therefore, the first two principal components were further evaluated. Table III presents the eigen values and factor loadings of these two principal components. The first principal component exhibited significant load based on the eigenvalues of NCA, CA, CCA, ICAB, ICAA and ICAC $(>0.8)$. Consequently, these six constituents were selected as representative variables for the antipyretic effect.

As presented in Table IV, the eigen values of the first two principal components were $>1$, and the contribution rate of the first principal component was $82.246 \%$. The results indicated that the first principal component could explain $82.246 \%$ of the anti-endotoxin activity of different species of LJT, LFH and LMH. The second principal component could explain $17.754 \%$, and the cumulative contribution rate of the first two principal components was $100 \%$. Therefore, the first two principal components were evaluated further. Table IV indicates their eigen values and factor loadings. The first principal component exhibited significant load based on the eigenvalues of the six constituents $(>0.8)$. Consequently, NCA, CA, CCA, ICAB, ICAA and ICAC, were selected as representative variables for anti-endotoxin activity.

Bivariate analysis(BA).Theantipyreticeffectandanti-endotoxin activity of these extracts are indicated in Figs. 3 and 4. Based
Table I. Quantitative analysis of the eight main chemical constituents from different species of LJT, LFH and LMH.

\begin{tabular}{llll}
\hline Compound & LJT $(\mathrm{mg} / \mathrm{g})$ & LFH $(\mathrm{mg} / \mathrm{g})$ & LMH $(\mathrm{mg} / \mathrm{g})$ \\
\hline NCA & $19.33 \pm 3.35$ & $11.05 \pm 0.34$ & $33.62 \pm 0.91$ \\
CA & $48.48 \pm 8.05$ & $19.81 \pm 0.60$ & $64.78 \pm 1.86$ \\
CCA & $26.05 \pm 4.27$ & $18.40 \pm 0.56$ & $47.49 \pm 1.53$ \\
SLA & $29.42 \pm 5.97$ & $29.94 \pm 0.95$ & 0.00 \\
SL & $12.92 \pm 2.32$ & $39.90 \pm 0.88$ & $27.50 \pm 1.21$ \\
ICAB & $22.28 \pm 13.85$ & $53.46 \pm 1.58$ & $27.68 \pm 0.78$ \\
ICAA & $13.85 \pm 2.28$ & $30.89 \pm 0.84$ & $17.22 \pm 0.32$ \\
ICAC & $24.58 \pm 4.22$ & $52.55 \pm 1.44$ & $25.60 \pm 0.54$ \\
\hline
\end{tabular}

LJT, Lonicera japonica Thunb.; LFH, Lonicerafulvotomentosa Hsu et S. C. Cheng; LMH, Loniceramacranthoides Hand.-Mazz.; NCA, neochlorogenic acid; $\mathrm{CA}$, chlorogenic acid; CCA, cryptochlorogenic acid; SLA, secologanic acid; SL, secoxyloganin; ICAB, isochlorogenic acid B; ICAA, isochlorogenic acid A; ICAC, isochlorogenic acid C.

on these results, a total of eight constituents significantly correlated with the concentration of cAMP $(\mathrm{P}<0.05)$, whereas seven constituents highly significantly correlated with the concentration of cAMP $(\mathrm{P}<0.01)$; a total of seven constituents significantly correlated with AVP levels $(\mathrm{P}<0.05)$, whereas four constituents highly significantly correlated with AVP levels $(\mathrm{P}<0.01)$. The constituents that significantly correlated with the concentrations of cAMP and AVP were NCA, CA, CCA, SL, ICAB, ICAA and ICAC (Fig. 3). These seven constituents exerted antipyretic effects. A total of eight constituents highly significantly correlated with ET levels $(\mathrm{P}<0.01)$. The constituents that significantly correlated with ET content were NCA, CA, CCA, SLA, SL, ICAB, ICAA and ICAC (Fig. 4). Therefore, these eight constituents were demonstrated to exert anti-endotoxin activity. The comprehensive findings of the present study verified that different species of LJT, LFH and LMH exhibited antipyretic effects and anti-endotoxin activities.

\section{Discussion}

To obtain a better chromatogram map, methanol-water, acetonitrile-water, methanol- $0.15 \%$ formic acid, acetonitrile- $0.15 \%$ formic acid, acetonitrile- $0.4 \%$ acetic acid, acetonitrile: Methanol (1:1)-0.4\% acetic acid and acetonitrile- $0.4 \%$ phosphoric acid were investigated and screened as mobile phases. The combination of acetic acid $(0.4 \%, \mathrm{v} / \mathrm{v})$-acetonitrile was the best mobile phase for separation and analysis. In the range of 190-400 $\mathrm{nm}$ full wavelength scanning, $254 \mathrm{~nm}$ was selected as optimal. The column temperature was set at 25,30 and $35^{\circ} \mathrm{C}$, respectively. A temperature of $35^{\circ} \mathrm{C}$ was selected. Flow rates of $0.8,1.0$ and $1.2 \mathrm{ml} / \mathrm{min}$ were also tested, and peak resolution of the samples was improved when the flow rate was $0.8 \mathrm{ml} / \mathrm{min}$. Lastly, injection volumes of 10,15 and $20 \mu \mathrm{l}$ were also compared, and peak resolution was optimized when the injection volume was $10 \mu \mathrm{l}$.

TCM has the characteristics of multi-component, multi-effect and collaborative integration. The composition of its components and the interactions between then is 
Table II. cAMP, AVP and ET content in rats from different experimental groups ( $\mathrm{n}=8$ rats/group).

\begin{tabular}{|c|c|c|c|c|}
\hline No & Group & cAMP content $(\mathrm{nmol} / \mathrm{ml})$ & AVP content (pg/ml) & ET content $(\mathrm{EU} / \mathrm{ml})$ \\
\hline 1 & LJT (6.75 g/kg) & $24.40 \pm 1.598^{\mathrm{a}, \mathrm{d}}$ & $23.28 \pm 2.12^{\mathrm{b}}$ & $164.13 \pm 12.98^{\mathrm{b}, \mathrm{c}}$ \\
\hline 2 & LJT (13.5 g/kg) & $21.85 \pm 0.669^{b}$ & $24.96 \pm 0.91^{\mathrm{b}, \mathrm{c}}$ & $146.62 \pm 10.03^{\mathrm{b}, \mathrm{c}}$ \\
\hline 3 & LJT (27 g/kg) & $19.73 \pm 1.084^{\mathrm{b}, \mathrm{c}}$ & $27.78 \pm 1.58^{\mathrm{b}}$ & $131.15 \pm 10.51^{\mathrm{b}, \mathrm{c}}$ \\
\hline 4 & LFT (6.75 g/kg) & $24.85 \pm 1.058^{\mathrm{a}}$ & $24.22 \pm 1.87^{\mathrm{b}, \mathrm{c}}$ & $178.34 \pm 13.83^{\mathrm{b}, \mathrm{c}}$ \\
\hline 5 & LFT (13.5 g/kg) & $22.16 \pm 0.934^{\mathrm{b}}$ & $25.88 \pm 2.59^{b, c}$ & $159.20 \pm 14.28^{\mathrm{b}, \mathrm{c}}$ \\
\hline 6 & LFT (27 g/kg) & $20.66 \pm 0.762^{\mathrm{b}, \mathrm{c}}$ & $27.20 \pm 3.08^{b, c}$ & $146.60 \pm 5.29^{\mathrm{b}, \mathrm{c}}$ \\
\hline 7 & LMT (6.75 g/kg) & $22.45 \pm 1.719^{\mathrm{b}}$ & $23.69 \pm 2.27^{\mathrm{b}, \mathrm{c}}$ & $153.23 \pm 12.93^{\mathrm{b}, \mathrm{c}}$ \\
\hline 8 & LMT (13.5 g/kg) & $20.63 \pm 1.068^{\mathrm{b}, \mathrm{c}}$ & $25.62 \pm 2.07^{\mathrm{b}, \mathrm{c}}$ & $138.38 \pm 6.33^{\mathrm{b}, \mathrm{c}}$ \\
\hline 9 & LMT (27 g/kg) & $18.71 \pm 1.553^{\mathrm{b}, \mathrm{c}}$ & $27.33 \pm 1.73^{\mathrm{b}, \mathrm{c}}$ & $122.82 \pm 10.44^{\mathrm{b}, \mathrm{c}}$ \\
\hline 10 & Model $(6.75 \mathrm{~g} / \mathrm{kg})$ & $25.95 \pm 1.175^{\mathrm{a}}$ & $17.88 \pm 3.26^{\mathrm{b}, \mathrm{c}}$ & $208.68 \pm 12.87^{\mathrm{c}}$ \\
\hline 11 & Positive $(6.75 \mathrm{~g} / \mathrm{kg})$ & $20.64 \pm 1.421^{\mathrm{b}}$ & $28.59 \pm 1.74^{\mathrm{b}, \mathrm{c}}$ & $143.78 \pm 9.94^{\mathrm{b}}$ \\
\hline 12 & Blank $(6.75$ g/kg) & $22.84 \pm 0.677^{b}$ & $22.92 \pm 1.83^{\mathrm{b}}$ & $102.80 \pm 8.23^{\mathrm{b}}$ \\
\hline
\end{tabular}

${ }^{\mathrm{a}} \mathrm{P}<0.05$ and ${ }^{\mathrm{b}} \mathrm{P}<0.01$ vs. model control group; ${ }^{\mathrm{c}} \mathrm{P}<0.01$ and ${ }^{\mathrm{d}} \mathrm{P}<0.05$ vs. blank control group. LJT, Lonicera japonica Thunb.; LFH, Lonicera fulvotomentosa Hsu et S. C. Cheng; LMH, Lonicera macranthoides Hand.-Mazz.; AVP, arginine vasopressin; ET, endotoxin.<smiles>O=C(O)C1C[C@](O)(C(=O)O)C[C@@H](O)[C@@H]1O</smiles>

NCA<smiles>O=C(/C=C/c1ccc(O)c(O)c1)O[C@@H]1C[C@@](O)(C(=O)O)C[C@@H](O)[C@H]1O</smiles>

CA<smiles>O=C(O)C1(O)C[C@H](O)[C@H](O)C[C@@]1(O)C(=O)O</smiles>

CCA<smiles>C=CC12CC(O)OC(=O)C1=COC2OC1OC(CO)[C@@H](O)C(O)C1O</smiles>

SLA<smiles>C=CC1C(O[C@@H]2O[C@H](CO)[C@@H](O)[C@H](O)C2O)OC=C(C(=O)OC)[C@H]1CC(=O)O</smiles>

SL

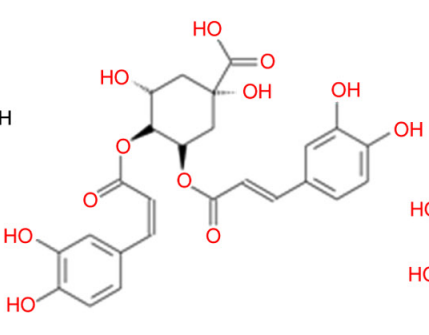

ICAB

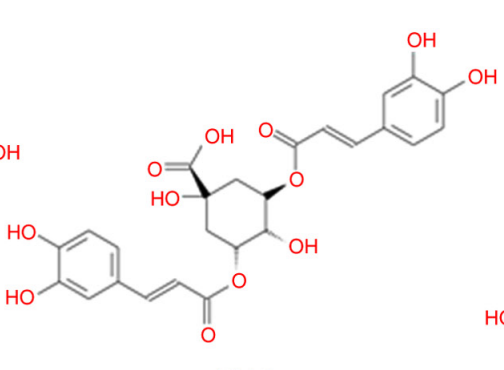

ICAA

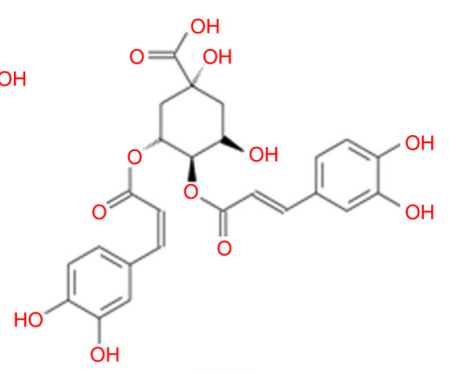

ICAC

Figure 2. Structures of the eight main chemical constituents identified in different species of Lonicera japonica Thunb., Lonicera fulvotomentosa Hsu et S. C. Cheng and Lonicera macranthoides Hand-Mazz. NCA, neochlorogenic acid; CA, chlorogenic acid; CCA, cryptochlorogenic acid; SLA, secologanic acid; SL, secoxyloganin; ICAB, isochlorogenic acid B; ICAA, isochlorogenic acid A; ICAC, isochlorogenic acid C.

complex. It is important to identify a suitable research method to determine the efficacy of TCM and its mechanism of action. At present, the most common research method is to determine pharmacodynamics and assess the correlation of its 'spectrum-effect' (19-29). Pearson's correlation coefficient was used in the current study to calculate the relative peak areas, which corresponded to the antipyretic effect and anti-endotoxin activity of the samples. Based on these results, different species of LJT, LFH and LMH exhibited antipyretic effects and anti-endotoxin activities. Correlation coefficients $>0.3$ or $<-0.3$ were considered significant $(\mathrm{P}<0.05)$, whereas correlation coefficients $>0.5$ or $<-0.5$ were considered highly significant $(\mathrm{P}<0.01)$. Based on the comprehensive results of PCA and BA, these eight constituents (NCA, CA, CCA, SLA,
SL, ICAB, ICAA and ICAC) were demonstrated to exert anti-endotoxin activity.

LC technology was used for the qualitative and quantitative analysis of eight main chemical constituents in different species of LJT, LFH and LMH. The present study illustrated how six constituents, namely NCA, CA, CCA, ICAB, ICAA and ICAC, were selected as representative variables for the antipyretic effect and anti-endotoxin activity. The constituents that significantly correlated with the concentration of cAMP and AVP were NCA, CA, CCA, SL, ICAB, ICAA and ICAC. The constituents that significantly correlated with ET levels were NCA, CA, CCA, SLA, SL, ICAB, ICAA and ICAC. Therefore, NCA, CA, CCA, ICAB, ICAA and ICAC were demonstrated to bethe key substances that mediate the 
Table III. Eigen values and corresponding percentages of the variables of antipyretic effect (top two principal components).

Initial eigen values

\begin{tabular}{lcccccrr}
\cline { 3 - 4 } Component & Total & Variance $(\%)$ & Cumulative $(\%)$ & & Total & Variance (\%) & Cumulative $(\%)$ \\
\hline 1 & 9.443 & 67.447 & 67.447 & 9.443 & 67.447 & 67.447 \\
2 & 4.557 & 32.553 & 100.000 & 4.557 & 32.553 & 100.000 \\
\hline
\end{tabular}

Table IV. Eigen values and corresponding percentages of the variables of anti-endotoxin activity (top two principal components).

Initial eigen values

\begin{tabular}{|c|c|c|c|c|c|c|}
\hline \multirow[b]{2}{*}{ Component } & & \\
\hline & Total & Variance $(\%)$ & Cumulative (\%) & Total & Variance $(\%)$ & Cumulative (\%) \\
\hline 1 & 9.047 & 82.246 & 82.246 & 9.047 & 82.246 & 82.246 \\
\hline 2 & 1.953 & 17.754 & 100.000 & 1.953 & 17.754 & 100.000 \\
\hline
\end{tabular}

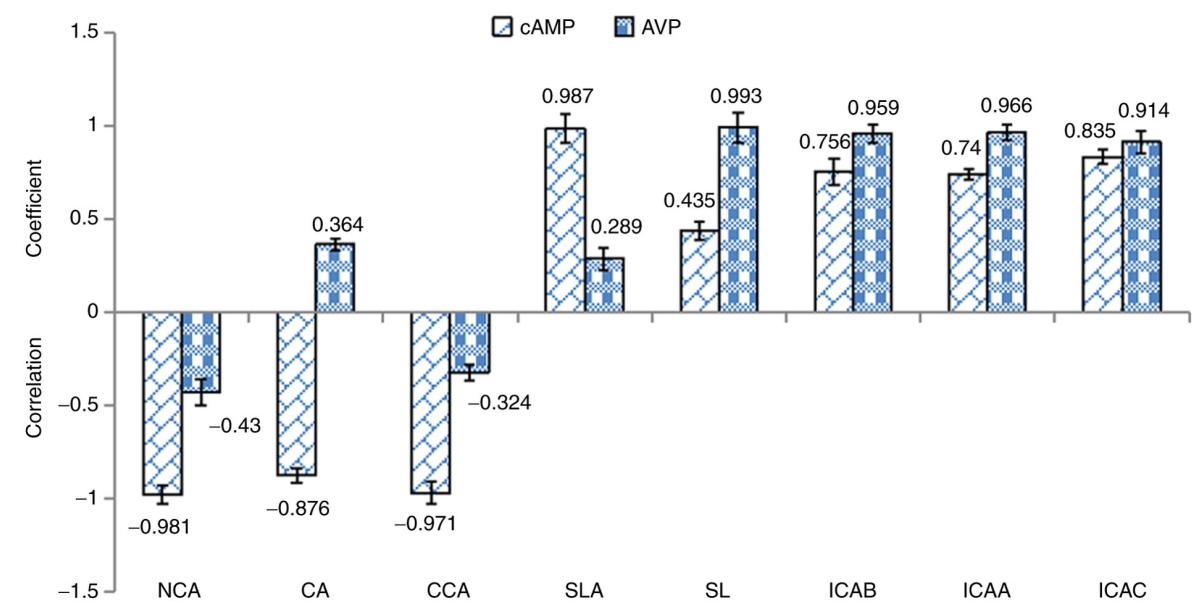

Figure 3. Bivariate analysis of eight main chemical constituents identified in different species of Lonicera japonica Thunb., Lonicera fulvotomentosa Hsu et S. C. Cheng and Loniceramacranthoides Hand-Mazz. with regards to the antipyretic effect. Correlation coefficients $>0.3$ or $<-0.3$ were considered significant $(\mathrm{P}<0.05)$, whereas correlation coefficients $>0.5$ or $<-0.5$ were considered highly significant $(\mathrm{P}<0.01)$. NCA, neochlorogenic acid; CA, chlorogenic acid; CCA, cryptochlorogenic acid; SLA, secologanic acid; SL, secoxyloganin; ICAB, isochlorogenic acid B; ICAA, isochlorogenic acid A; ICAC, isochlorogenic acid C; AVP, arginine vasopressin.

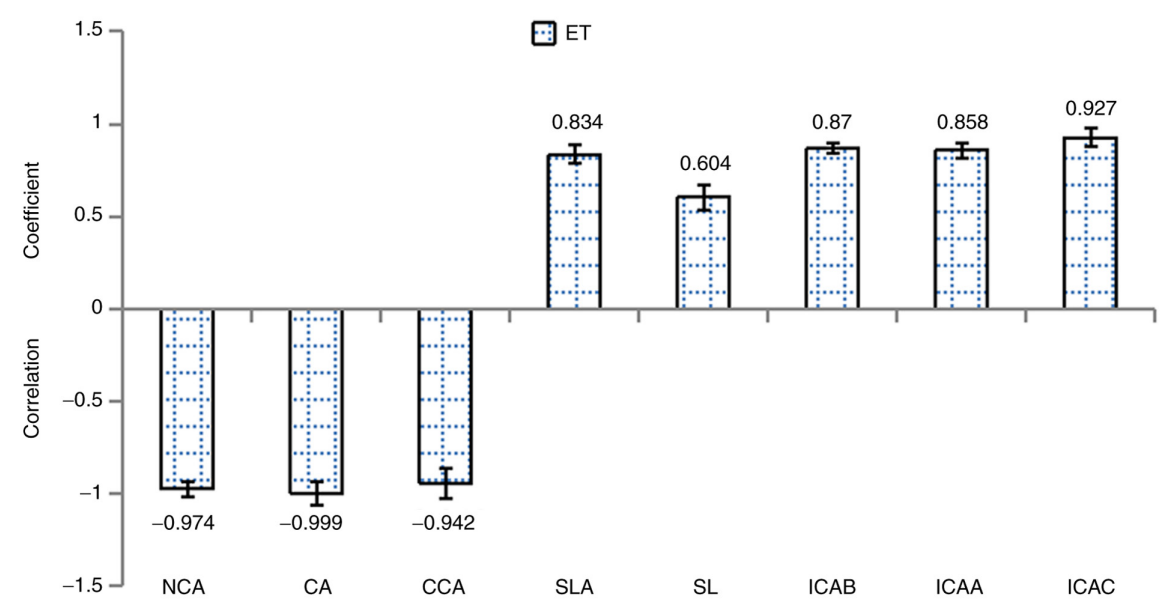

Figure 4. Bivariate analysis of the eight main chemical constituents identified in different species of Lonicera japonica Thunb., Lonicerafulvotomentosa Hsu et S. C. Cheng and Loniceramacranthoides Hand-Mazz. with regards to anti-endotoxin activity. Correlation coefficients $>0.3$ or $<-0.3$ were considered significant ( $\mathrm{P}<0.05$ ), whereas correlation coefficients $>0.5$ or $<-0.5$ were considered highly significant $(\mathrm{P}<0.01)$. NCA, neochlorogenic acid; $\mathrm{CA}$, chlorogenic acid; CCA, cryptochlorogenic acid; SLA, secologanic acid; SL, secoxyloganin; ICAB, isochlorogenic acid B; ICAA, isochlorogenic acid A; ICAC, isochlorogenic acid C; ET, endotoxin. 
antipyretic effect and anti-endotoxin activity of different species of Lonicera japonica.

\section{Acknowledgements}

Not applicable.

\section{Funding}

The present project was supported by the Science and Technology Project of Guizhou Province [grant no. QKHZC (2016) 2909], Guizhou Discipline Construction Project [grant no. GNYL (2017) 008], the Guizhou Province 'Hundred' Innovative Talents Project [grant no. QKHRC (2015) 4032] and the Guizhou University of Traditional Chinese Medicine Construction Project of Undergraduate Teaching Engineering [grant no. GZYJXGCHZ (2019) 54].

\section{Availability of data and materials}

The datasets used and/or analyzed during the current study are available from the corresponding author on reasonable request.

\section{Authors' contributions}

JXD wrote the manuscript and analyzed data. YZ designed the experiments. WNY established and evaluated the fingerprint. WPL carried out the ELISA experiments. HS and JXL performed the animal experiments. CLT recorded and analyzed the experimental data. CL and XWL analyzed the data. All authors read and approved the final manuscript.

\section{Ethics approval and consent to participate}

All experimental procedures were reviewed and approved by The Animal Ethics Committee of Guizhou University (approval no. GZU-2019-11).

\section{Patient consent for publication}

Not applicable.

\section{Competing interests}

The authors declare that they have no competing interests.

\section{References}

1. Wang Z, Xia Q, Liu X, Liu W, Huang W, Mei X, Luo J, Shan M, Lin R, Zou D and Ma Z: Phytochemistry, pharmacology, quality control and future research of Forsythia suspensa (Thunb.) Vahl A review. J Ethnopharmacol 210: 318-339, 2018.

2. Shu Y, Liu Z, Zhao S, Song Z, He D, Wang M, Zeng H, Lu C, $\mathrm{Lu} \mathrm{A}$ and Liu Y: Integrated and global pseudotargeted metabolomics strategy applied to screening for quality control markers of Citrus TCMs. Anal Bioanal Chem 409: 4849-4865, 2017.

3. Zhuo L, Peng J, Zhao Y, Li D, Xie X, Tong L and Yu Z: Screening bioactive quality control markers of QiShenYiQi dripping pills based on the relationship between the ultra-high performance liquid chromatography fingerprint and vascular protective activity. J Sep Sci 40: 4076-4084, 2017.

4. Sander MH, Joost F, Ger TR, Ronald JAW, Wietse K and Hans RW: Temperature dependence of mutant mevalonate kinase activity as a pathogenic factor in hyper- $\operatorname{IgD}$ and periodic fever syndrome. Hum Mol Genet 11: 3115-3124, 2002.
5. Kailash $P$ and Shuchita T: Therapeutic interventions for advanced glycation-end products and its receptor- mediated cardiovascular disease. Curr Pharm Des 23: 937-943, 2017.

6. Jaroslawska J, Chabowska-Kita A, Kaczmarek MM and Kozak LP: Npvf: Hypothalamic biomarker of ambient temperature independent of nutritional status. PLoS Genet 11: e1005287, 2015.

7. do Nascimento AF, Thompsom B, Dell'Armelina Rocha PR Kirychuk S, Bernardi MM and Felicio LF: Hyperprolactinemia impaired the effects of lipopolysaccharide on both body temperature and sickness behavior in virgin female rats. Neuroimmunomodulation 26: 285-291, 2019.

8. Sylvia KE and Demas GE: Acute intraperitoneal lipopolysaccharide influences the immune system in the absence of gut dysbiosis. Physiol Rep 6: e13639, 2018.

9. Hussain MS, Azam F, Ahamed KF, Ravichandiran V and Alkskas I: Anti-endotoxin effects of terpenoids fraction from Hygrophila auriculata in lipopolysaccharide-induced septic shock in rats. Pharm Biol 54: 628-636, 2016.

10. Malan M, Serem JC, Bester MJ, Neitz AW and Gaspar AR: Anti-inflammatory and anti-endotoxin properties of peptides derived from the carboxy-terminal region of a defensin from the tick Ornithodoros savignyi. J Pept Sci 22: 43-51, 2016.

11. Cavaillon JM: Exotoxins and endotoxins: Inducers of inflammatory cytokines. Toxicon 149: 45-53, 2018.

12. Saleh E, Moody MA and Walter EB: Effect of antipyretic analgesics on immune responses to vaccination. Hum Vaccin Immunother 12: 2391-402, 2016.

13. Elahe M, Johan LÅN, Kiseko S, Anders B, Peter MZ, David E, Edward DH and Anders B: The antipyretic effect of paracetamol occurs independent of transient receptor potential ankyrin 1-mediated hypothermia and is associated with prostaglandin inhibition in the brain. FASEB J 32: 5751-5759, 2018.

14. Jennifer L and Hazel MW: Antipyretic drugs in patients with fever and infection: Literature review. Br J Nurs 28: 610-618, 2019.

15. Muhammad TI: Antipyretic effect of phytol, possibly via 5KIRdependent COX-2 inhibition pathway. Inflammopharmacology 27: 857-862, 2019.

16. Lü SW, Su H, Sun S, Guo YY, Liu T, Ping Y and Li YJ: Isolation and characterization of nanometre aggregates from a Bai-Hu-Tang decoction and their antipyretic effect. Sci Rep 8: 12209, 2018.

17. Wang XM, Xu WJ, Xu LK, Song S, Xing XF and Luo JB: Antipyretic effect of Herba Ephedrae-Ramulus Cinnamomi herb pair on yeast-induced pyrexia rats: A metabolomics study. Chin J Integr Med 24: 676-682, 2018.

18. Choi SJ, Moon S, Choi UY, Chun YH, Lee JH, Rhim JW, Lee J, Kim HM and Jeong DC: The antipyretic efficacy and safety of propacetamol compared with dexibuprofen in febrile children: A multicenter, randomized, double-blind, comparative, phase 3 clinical trial. BMC Pediatr 18: 201, 2018.

19. Ding JX,LiWL,Hu Y,Song H,SunXMand Ji YB: Characterization of estrogenic active ingredients in Cuscutachinensis Lam. based on spectral characteristics and high-performance liquid chromatography/quadrupole time-of-flight mass spectrometry. Mol Med Rep 19: 1238-1247, 2019.

20. Hernaez R and Thrift AP: High negative predictive value, low prevalence, and spectrum effect: Caution in the interpretation. Clin Gastroenterol Hepatol 15: 1355-1358, 2017.

21. Liang J, Chen Y, Ren G, Dong W, Shi M, Xiong L, Li J, Dong J, Li F and Yuan J: Screening hepatotoxic components in Euodia rutaecarpa by UHPLC-QTOF/MS based on the spectrum-toxicity relationship. Molecules 22: E1264, 2017.

22. Chopard G, Puyraveau M, Binetruy M, Meyer A, Vandel P, Magnin E, Berger E, Galmiche J and Mauny F: Spectrum effect and spectrum bias in the screening test performance for amnestic mild cognitive impairment: What are the clinical implications? J Alzheimers Dis 48: 385-393, 2015.

23. Xu GL, Xie M, Yang XY, Song Y, Yan C, Yang Y, Zhang X, Liu ZZ, Tian YX, Wang Y, et al: Spectrum-effect relationships as a systematic approach to traditional chinese medicine research: Current status and future perspectives. Molecules 19: 17897-17925, 2014.

24. Zheng Q, Zhao Y, Wang J, Liu T, Zhang B, Gong M, Li J, Liu H, Han B, Zhang Y, et al: Spectrum-effect relationships between UPLC fingerprints and bioactivities of crude secondary roots of Aconitum carmichaelii Debeaux (Fuzi) and its three processed products on mitochondrial growth coupled with canonical correlation analysis. J Ethnopharmacol 153: 615-623, 2014. 
25. Li W, Sun X, Liu B, Zhang L, Fan Z and Ji Y: Screening and identification of hepatotoxic component in Evodia rutaecarpa based on spectrum-effect relationship and UPLC-Q-TOFMS. Biomed Chromatogr 30: 1975-1983, 2016.

26. Shi Z, Liu Z, Liu C, Wu M, Su H, Ma X, Zang Y, Wang J, Zhao Y and Xiao X: Spectrum-effect relationships between chemical fingerprints and antibacterial effects of Lonicerae Japonicae Flos and Lonicerae Flos base on UPLC and microcalorimetry. Front Pharmacol 7: 12, 2016

27. Chen Y, Yu H, Wu H, Pan Y, Wang K, Liu L, Jin Y and Zhang C: Tracing novel hemostatic compounds from heating products of total flavonoids in Flos Sophorae by spectrum-effect relationships and column chromatography. J Sep Sci 38: 1691-1699, 2015

28. Liu X, Wang XL, Wu L, Li H, Qin KM, Cai H, Pei K, Liu T and Cai BC: Investigation on the spectrum-effect relationships of Da-Huang-Fu-Zi-Tang in rats by UHPLC-ESI-Q-TOF-MS method. J Ethnopharmacol 154: 606-612, 2014.

29. Xie RF, Zhou X, Shi ZN, Li YM and Li ZC: Study on spectrum-effect relationship of rhizome Rhei, cortex Magnoliae Officinalis, fructus Aurantii Immaturus and their formula. J Chromatogr Sci 51: 524-532, 2013.

30. Li WL, Ding JX, Bai J, Hu Y, Song H, Sun XM and Ji YB: Research on correlation of compositions with oestrogenic activity of Cistanche based on LC/QTOF-MS/MS technology. Open Chem 17: 1-12, 2019.

31. Li WL, Ding JX, Liu BM, Zhang DL, Song H, Sun XM, Liu GY, Wang JY and Ji YB: Phytochemical screening and estrogenic activity of total glycosides of Cistanchedeserticola. Open Chem 17: 279-287, 2019.

32. Li YK, Li W, Fu CM, Song Y and Fu Q: Lonicerae Japonicae Flos and Lonicerae Flos: A systematic review of ethnopharmacology, phytochemistry and pharmacology. Phytochem Rev: 1-61, 2019.

33. National Pharmacopoeia Committee. Pharmacopoeia of the people's Republic of China. Volume I. Beijing: China Medical Science and Technology Press, 221, 2015.

34. Zhang F, Shi PL, Liu HY, Zhang YQ Yu X, Li J and Pu GB A simple, rapid, and practical method for distinguishing Lonicerae Japonicae Flos from Lonicerae Flos. Molecules 24 $3455,2019$.
35. Zhang X, Guo Q and Yu BY: Rapid quantitative analysis of adulterant Lonicera species in preparations of Lonicerae Japonicae Flos. J Sep Sci 38: 4014-4020, 2015.

36. Qian WJ, Kang A, Peng LX, Xie T, Ji JJ, Zhou W, Shan JJ and Di LQ: Gas chromatography-mass spectrometry based plasma metabolomics of H1N1-induced inflammation in mice and intervention with Flos Lonicerae Japonica-Fructus Forsythiae herb pair. J Chromatogr B Analyt Technol Biomed Life Sci 1092: 122-130, 2018

37. Yan WN: Study on fingerprint of Lonicera japonica flos and loniceraeflos in guizhou province. Guizhou University. Master Thesis, 2018

38. Niu F, Xu X,Zhang R, Sun L, Gan N and Wang A: Ursodeoxycholic acid stimulates alveolar fluid clearance in LPS-induced pulmonary edema via ALX/cAMP/PI3K pathway. J Cell Physiol 234: 20057-20065, 2019.

39. Hitoshi S, Makoto K, Hideo O, Toshitaka N and Yoichi U: Regulatory mechanism of the arginine vasopressin-enhanced green fluorescent protein fusion gene expression in acute and chronic stress. Peptides 30: 1763-1770, 2009.

40. Bossola M, Di Stasio E, Sanguinetti M, Posteraro B, Antocicco M, Pepe G, Mello E, Bugli F and Vulpio C: Serum endotoxin activity measured with endotoxin activity assay is associated with serum interleukin-6 levels in patients on chronic hemodialysis. Blood Purif 42: 294-300, 2016.

41. Chatkupt TT, Libal NL, Mader SL, Murphy SJ and Saunders KE: Effect of continuous trio breeding compared with continuous pair breeding in 'Shoebox' Caging on measures of reproductive performance in estrogen receptor knockout mice. J Am Assoc Lab Anim Sci 57: 328-334, 2018.

(i) $($ ) This work is licensed under a Creative Commons Attribution-NonCommercial-NoDerivatives 4.0 International (CC BY-NC-ND 4.0) License. 